\title{
Hodges-Lehmann scale estimator for Cauchy distribution
}

\author{
O.Y. Kravchuk*, P.K.Pollett \\ SLCAFS, MASCOS \\ University of Queensland \\ Hartley Teakle Bld, University of Queensland, \\ Brisbane, QLD, 4072, Australia; \\ *o.kravchuk@uq.edu.au; \\ phone: (61 7) 33652171; fax: (61 7) 33651177
}

topic area: rank tests, Hodges-Lehamnn estimator, scale estimation, hyperbolic secant distribution

\begin{abstract}
We draw here on the relation between the Cauchy and hyperbolic secant distributions to prove that the MLE of the scale parameter of Cauchy distribution is log-normally distributed and to study the properties of a Hodges-Lehmann type estimator for the scale parameter. This scale estimator is slightly biased but performs well even on small samples regardless of the location parameter. The asymptotic efficiency of the estimator is $98 \%$.
\end{abstract}

\section{Introduction}

The Cauchy distribution is a heavy-tail error distribution common in physics, econometrics and engineering (refer to a brief overview in [22]). This distribution often results from ratios of random variables [1], a textbook example being the ratio of two independent standard normal variables. The Cauchy distribution is a location-scale symmetric distribution (denoted $C(\mu, \sigma)$ throughout the text); the standard 
Cauchy distribution has a location of zero and a scale of one $(C(0,1))$.

The problem of estimation of the location and scale parameters of Cauchy distribution is challenging. The simple moment estimators do not exist, and alternative estimators are often computationally problematic. Many estimation approaches have been suggested in the literature: joint maximum (and modified maximum) likelihood $[10,25,26]$, order statistics [5, 24], window estimates [12], empirical characteristic function [16, 2, 20], minimum distance [3], Bayesian [15] and linear rank estimators $[4,17]$. Such a multitude of estimation procedures is an attempt to balance the ease of computations with the efficiency, robustness and consistency of estimators.

The standard Cauchy variable has an interesting property that the $\log$-transformation of its absolute value $(\ln |C(0,1)|)$ follows a symmetric location-scale distribution - the standard hyperbolic secant distribution $(\operatorname{HSD}(\mu, \sigma)$ hereafter, the standard $\operatorname{HSD}$ is $\operatorname{HSD}(0,1))[18,17]$. It was shown in [17] that the optimal location rank estimator for the HSD is a fully efficient scale estimator for the Cauchy distribution with the location parameter of zero. Moreover, it was also proven there that the Hodges-Lehmann estimator calculated on the log-transformed absolute values from $C(0, \sigma)$ is more than $98 \%$ efficient for the scale parameter $\sigma$. In this paper, we study the asymptotic and small-sample properties of that estimator and expand its application to the case of non-centred Cauchy distribution $C\left(\mu_{0}, \sigma\right)$ whose location parameter $\mu_{0} \neq 0$ may or may not be known. Additionally, we add here to the knowledge about the small-sample and asymptotic behaviour of the maximum likelihood estimator of the scale parameter and correct the current misconception that its asymptotic dustribution is normal [13].

The paper is organised in the following way. In Section 2, we give a proof that the Cauchy maximum likelihood estimator of scale is asymptotically log-normal. In Section 3, we discuss the distribution of the Hodges-Lehmann estimator on the log-transformed data for $C(\mu, \sigma)$, where $\mu$ is known. In Section 4 , we modify the HodgesLehmann estimator for the case of unknown location parameter. In Section 5, we compare the small-sample and asymptotic performances of the maximim likelihood and Hodges-Lehmann estimators. In Section 6 , we step the reader through a real-life example for the scale estimation procedures introduced in the paper and conclude the study. 


\section{Asymptotic distribution of the MLE of scale when the location is known}

Let us consider a random variable $X$ that follows a centred Cauchy distribution, $C(0, \sigma)$, of the density function $f$ :

$$
f(x)=\frac{1}{\pi \sigma} \frac{1}{1+(x / \sigma)^{2}}, \sigma>0 .
$$

The natural logarithm of the absolute value of this Cauchy variable follows the hyperbolic secant distribution, $Y=\ln |X| \sim H S D(\ln \sigma, 1)$, of the density function $g$ :

$$
g(y)=\frac{1}{\pi} \operatorname{sech}(y-\ln \sigma) .
$$

The location information number of the HSD is $1 / 2$ [17].

The maximum likelihood estimator of the scale parameter for the Cauchy distribution of a known location parameter $\mu_{0}$ has the following simple definition:

$$
\operatorname{MLE}(\sigma)=\left(\hat{\sigma}: \sum \frac{\hat{\sigma}^{2}}{\left(x-\mu_{0}\right)^{2}+\hat{\sigma}^{2}}=\frac{n}{2}\right) .
$$

The solution to the maximum likelihood estimator of the scale parameter exists for $n>2$ and is unique [6].

The exact percentiles of the ratio $\operatorname{MLE}(\sigma) / \sigma$, derived by MonteCarlo simulations, are tabulated for sample sizes between 10 and 100 in [10]. It was also suggested there that the asymptotic distribution of the ratio $\operatorname{MLE}(\sigma) / \sigma$ follows the normal distribution of mean 1 and variance $2 / n$. On the other hand, it was informally demonstrated in [15] that the distribution of the ratio is positively skewed for large samples. We argue here that the asymptotic distribution of the ratio is lognormal, with the mean and variance of the natural logarithm of the variate being 0 and $2 / n$ correspondingly. A proof for this is outlined below.

Proposition 1: The asymptotic distribution of the MLE of scale for a sample of $n$ observations from a Cauchy distribution with any known location parameter $\mu_{0}, C\left(\mu_{0}, \sigma\right)$, is lognormal $(\ln \sigma, 2 / n)$. 
Consider the log-likelihood function of location for a hyperbolic secant distribution of a location parameter $\ln \sigma$ and the unit scale parameter, $Y \sim H S D(\ln \sigma, 1)$ :

$$
\left.\ln L=-n \ln \pi-\sum \ln \cosh (y-\ln \sigma)\right), \sigma>0 .
$$

Hence

$$
\frac{\partial \ln L}{\partial \ln \sigma}=\sum\left(1-\frac{2}{(\exp (y-\ln \sigma))^{2}+1}\right),
$$

and the maximum likelihood equation for $\ln (\sigma)$ is

$$
\sum\left(\frac{\exp (\ln \sigma)^{2}}{\exp (y)^{2}+\exp (\ln \sigma)^{2}}\right)=\frac{n}{2}
$$

Recalling that $Y=\ln \left|X-\mu_{0}\right|$ is distributed as $\operatorname{HSD}(\ln \sigma, 1)$ if and only if $X \sim C\left(\mu_{0}, \sigma\right)$, the maximum likelihood equation above may be expressed in terms of $X$ as

$$
\sum \frac{\sigma^{2}}{\sigma^{2}+\left(x-\mu_{0}\right)^{2}}=\frac{n}{2}
$$

which is exactly the MLE equation for the scale parameter $\sigma$ of Cauchy, (1). By Proposition 1 in [27], the MLE of the location parameter of HSD is asymptotically unbiased and normally distributed with the variance of $2 / n$. Therefore, the MLE of scale for the corresponding Cauchy $C\left(\mu_{0}, \sigma\right)$, where $\mu_{0}$ is known, is $\operatorname{lognormal}(\ln \sigma, 2 / n)$. This ends the proof.

The expected value of the MLE of scale is $E(M L E(\sigma))=\sigma \exp (1 / n)$, hence the estimator is positively biased. The bias is linear in the scale parameter and diminishes quickly as $n$ increases. The variance of this biased estimator is proportional to the square of the true value of the scale parameter, $\operatorname{Var}(\operatorname{MLE}(\sigma))=\sigma^{2} \exp (2 / n)(\exp (2 / n)-1)$.

These results clarify several computational observations about the properties of the MLE presented elsewhere. In particular, the bias was estimated as $E(\operatorname{MLE}(\sigma))-\sigma \cong \sigma / n$ in [19], which corresponds to the first-order expansion of its exact definition, $\exp (1 / n)-1=$ $1 / n+1 /\left(2 ! n^{2}\right)+\cdots$. Let us also draw the reader's attention to that, for samples larger than $n=20$, the exact percentiles of $\operatorname{MLE}(\sigma) / \sigma$ (Table 3 , [10]) agree better with the log-normal asymptotic distribution than with the normal $(1,2 / \mathrm{n})$ suggested earlier in [10]. For example, for 
$n=100$, the exact $90 \%$-confidence interval of the ratio $(0.790,1.267)$ is much closer to the approximate log-normal $(0.792,1.262)$ defined by Proposition 1 in this paper than the approximate normal $(0.768$, 1.232) proposed in [10].

The straightforward connection between the Cauchy distribution and the HSD allows us to transform easily a location estimator for the HSD into a scale estimator for the Cauchy distribution. In the following section, we discuss the properties of one of the most common location rank estimators, the Hodges-Lehmann estimator, in application to the scale estimation problem for the Cauchy distribution.

\section{Hodges-Lehmann estimator of scale when the location is known}

There exists an optimal linear rank estimator of scale for Cauchy distribution. It was reported elsewhere that the second component of the one-sample Cramer-von Mises statistic is fully efficient under the scale alternative for the centred Cauchy distribution $[3,8]$. Additionally, it was shown that the optimal scores for the rank estimator of location for the $\operatorname{HSD}(\mu, 1)$ correspond to the second component of the Cramer-von Mises statistic [17]. The convergence, distributional and robustness properties of the optimal rank estimator for the HSD were also discussed in [17], where it was pointed out that that asymptotically fully efficient rank estimator does not have a closed form solution and has to be found iteratively. On the other hand, the Hodges-Lehmann estimator is $98.6 \%$ efficient for the HSD, and can be easily and uniquely calculated. The asymptotic properties of the Hodges-Lehmann estimator are also well-known. For the HSD, this estimator is asymptotically unbiased and normally distributed with the variance of $\pi^{4} /(48 n)$ [17].

Hodges-Lehmann estimator is defined as a half of the median of $n(n+1) / 2$ pairwise sums of observations [14]. For a log-transformed centred Cauchy, $C(0, \sigma)$, the corresponding estimator is thus defined as

a half of the median of $n(n+1) / 2$ logarithms of the absolute values of pairwise products of the Cauchy observations; let us call this estimator 
$\log \operatorname{HLE}(\sigma)$ :

$$
\log \operatorname{HLE}(\sigma)=\frac{1}{2} \operatorname{med}\left(\ln \left|X_{i} X_{j}\right|\right), 1 \leq i, j \leq n, i \leq j .
$$

As has been mentioned earlier, the expected value of the $\log \operatorname{HLE}(\sigma)$ is $\ln \sigma$ and its asymptotic variance is $2 /\left(n\left(\pi^{2} / 4 \sqrt{6}\right)^{2}\right) \approx 2 /(0.986 n)$. The estimator is asymptotically normally distributed.

Let us define a back-transformed $\log \operatorname{HLE}(\sigma)$ (denoted $\operatorname{HLE}(\sigma)$ hereafter) simply as

$$
\operatorname{HLE}(\sigma)=\exp (\log H L E(\sigma)) .
$$

For Cauchy data, the HLE $(\sigma)$ follows the corresponding log-normal distribution, lognormal $\left(\ln \sigma, 2 /\left(n\left(\pi^{2} / 4 \sqrt{6}\right)^{2}\right)\right)$. The expected value and variance of this back-transformed scale estimator are:

$$
\mathrm{E}(\operatorname{HLE}(\sigma))=\sigma \sqrt{\exp [\operatorname{Var}(\log \operatorname{HLE}(\sigma))]},
$$

and

$$
\operatorname{Var}(\operatorname{HLE}(\sigma))=(\exp [\operatorname{Var}(\log H L E(\sigma))]-1) \exp [\operatorname{Var}(\log H L E(\sigma))] \sigma^{2} .
$$

The $\operatorname{HLE}(\sigma)$ is a biased estimator. The asymptotic estimation of the bias is

$E(\operatorname{HLE}(\sigma))-\sigma=\sigma\left(2 \exp \left(\pi^{2} /(4 \sqrt{6 n})\right)-1\right) \approx \sigma(\exp (1 /(0.986 n)-1)$.

This correction for bias is similar to that for the MLE. The magnitude of the correction depends on the sample size as well as the scale parameter itself. The bias correction is between $10 \%$ and $1 \%$ for samples with $10-100$ observations and between $1 \%$ and $0.5 \%$ for $100-200$ observations.

If the true location parameter of Cauchy is not known, the logHLE is not consistent for $\ln \sigma$. Some further adjustment to the procedure has to be done in order to account for an unknown location parameter.

\section{Hodges-Lehmann estimator of scale when the location parameter is unknown}

Let us start with deriving the distribution of the log-transform of a non-centred Cauchy distribution. 
Proposition 2: The natural logarithm of the absolute value of a non-centred Cauchy $C(\mu \neq 0, \sigma)$ follows a symmetric distribution with the expected value being a function of both $\mu$ and $\sigma, \ln \sqrt{\mu^{2}+\sigma^{2}}$.

Let $X \sim C(0, \sigma)$, then for any $\mu \neq 0, X+\mu \sim C(\mu, \sigma)$. The distribution function of $Y=\ln |X+\mu|$ is easy to derive if we notice first that

$$
\operatorname{Pr}(\ln |X+\mu| \leq y)=\operatorname{Pr}(X \leq \exp (y)-\mu)-\operatorname{Pr}(X \leq-\exp (y)-\mu),
$$

The expression for the density function $g$ of $Y$ is immediate:

$$
g(y)=\frac{\exp (y)}{\pi \sigma}\left(\frac{1}{1+(\exp (y)-\mu)^{2} / \sigma^{2}}+\frac{1}{1+(\exp (y)+\mu)^{2} / \sigma^{2}}\right) .
$$

Substituting $w=\exp (y)$ and using the Cauchy's residue theorem for complex variable, it is easy to show that the expected value of that distribution is

$$
\mu_{y}=2 \frac{\sigma}{\pi} \int_{0}^{\infty} \ln (w) /\left(\sigma^{2}+(w-\mu)^{2}\right) d w=\ln \left(\sqrt{\mu^{2}+\sigma^{2}}\right) .
$$

It is only left to show that

$$
g(y)=g\left(2 \mu_{y}-y\right)
$$

which is easy to see if we notice that

$$
\frac{\left(\mu^{2}+\sigma^{2}\right) \exp (y)}{\exp (y)^{2}+\left(\mu^{2}+\sigma^{2} \pm \mu \exp (y)\right)^{2} / \sigma^{2}}=\frac{\exp (y)}{1+(\exp (y) \pm \mu)^{2} / \sigma^{2}} .
$$

This ends the proof.

It is obvious that the HLE for a non-centred Cauchy sample is not consistent with $\ln \sigma$.

Several remedies have been proposed for estimating the scale parameter for a location-scale distribution, whose location parameter is not known. One approach is to shift the observations by a consistent and robust estimate of the location parameter [11]. In this section, we demonstrate that it is sufficient to adjust a random Cauchy sample by its sample median prior to taking the logarithm of the absolute values of observations and computing the Hodges-Lehmann estimator 
of scale. One can immediately notice that for samples with an odd number of observations, subtracting the median would cause the illdefined case of $\ln (0)$. To avoid this problem, for odd sample sizes, we suggest using the average of the values adjacent to the median (dropping one observation at random could be another option).

Our choice of the median is governed by the following: the sample median is unbiased, easy to derive, robust and about $80 \%$ efficient asymptotically for the Cauchy distribution. Additionally, for the purposes of this paper, we are not interested in jointly estimating the location and scale parameters but rather in the scale parameter alone; the value of the median is thus of little interest, and is only used as a means for achieving the HSD-like shape of the distribution of the log-transformed sample.

The median-adjusted logHLE (denoted logHLEM hereafter) is defined for $X \sim C(\mu, \sigma)$ as follows:

$$
\log \operatorname{HLEM}(\sigma)=\frac{1}{2} \operatorname{med}\left(\ln \left|\left(X_{i}-m\right)\left(X_{j}-m\right)\right|, 1 \leq i, j \leq n, i \leq j,\right.
$$

where $m$ is the sample median.

The variance of the sample median of Cauchy distribution is finite for samples $n \geq 2$, and for large samples it is $\pi^{2} /(4 n)$ [7]. Therefore, asymptotically, the median-adjusted estimator converges to the case of known location parameter; the asymptotic distribution of the estimator is thus normal $\left(\ln \sigma, 2 /\left(n\left(\pi^{2} / 4 \sqrt{6}\right)^{2}\right)\right.$. For small samples, we would expect the variance of $\operatorname{logHLEM}$ to be larger than that of $\operatorname{logHLE}$ as we introduce extra variation by subtracting the sample median.

In summary, the back-transformed estimator HLEM $=\exp (\log \operatorname{HLEM}(\sigma))$ is asymptotically log-normally distributed, slightly biased, has a larger variance for small samples, but is comparable to the HLE for large samples. This is also illustrated in Figure 1.

\section{Performances of the estimators for small and large samples}

There are two special cases of the MLE of scale, which are worth mentioning here. The closed form solutions exist for samples of three 
and four observations $[9,21]$. For these sample sizes, the closed form expressions of the $\log \mathrm{HLE}(\sigma)$ are also straightforward. We performed a small simulation study in the R-project [23] on the properties of the estimators. The study was based on random samples of sizes 3 or 4 generated with the rcauchy() function. Below, we give the explicit expressions for the MLE and logHLE for samples of 3 and 4 and briefly discuss their behaviour.

Let three random observations $(x, y$ and $z)$ from a Cauchy distribution be sorted, $x<y<z$. The MLE of scale is expressed as

$$
\operatorname{MLE}(\sigma)=\sqrt{3} \frac{(z-y)(z-x)(y-x)}{(z-y)^{2}+(z-x)^{2}+(y-x)^{2}},
$$

or in a slightly re-arranged form,

$$
\operatorname{MLE}(\sigma)=\frac{\sqrt{3}}{2}\left(\frac{1}{z-y}+\frac{1}{y-x}-\frac{1}{z-x}\right)^{-1} .
$$

From the definitions above, it is immediate that the MLE is locationinvariant. The behaviour of the estimator is not consistent with its asymptotic distribution. Our simulations suggest that the exact MLE is unbiased. The estimator demonstrates extreme variability, and we could not find a reliable estimate of its variance numerically in our computational study. The log-transformation fails to normalise the exact distribution of the MLE, with the Anderson-Darling test rejecting the hypothesis of normality, at the $5 \%$ significance, in about 350 from 500 runs (the ad.test() function in the nortest package in $\mathrm{R}$, each run contained $100 \ln$ (MLE) calculated for $n=3$ Cauchy random samples).

For the same three observations $(x, y, z)$, let the logarithms of their absolute values $(a, b, c)$ be sorted, $a<b<c$. The logHLE of scale is expressed as

$$
\log \mathrm{HLE}(\sigma)=\frac{2 b+a+c}{4} .
$$

The logarithms of the absolute values follow $\operatorname{HSD}(\ln \sigma, 1)$, whose variance is $\pi^{2} / 4$ [17]. The lower boundary of the variance of the logHLE is thus $3 \pi^{2} / 32$. The distribution of the estimator is more normal than the distribution of $\ln (\mathrm{MLE})$, although the normality is not yet satisfied at the nominal level. The Anderson-Darling test only rejected, at the $5 \%$ significance, the hypothesis of normality in about 110 out 
of 500 runs (100 logHLE each). The back-transformed estimator of scale, $\operatorname{HLE}(\sigma)$ is positively biased. The asymptotic estimation of the bias factor is $\sqrt{\exp \left(3 \pi^{2} / 32\right)}=1.588$, which is close to an empirical estimate of 1.52 we derived numerically. In our computational study, the variance of the HLE (with the bias-correction factor 1.52) was estimated to be about 1.77, which matches well its lower boundary of 1.66 under the lognormal approximation.

The variance of the minimum and maximum is unbounded for a Cauchy sample, and the median-adjusted estimator, logHLEM, is not defined in the case of $n=3$.

For the sample size $n=4$, let four random observations be sorted, $w<x<y<z$. The MLE of scale is expressed as

$$
\operatorname{MLE}(\sigma)=\frac{\sqrt{(z-y)(y-x)(x-w)(z-w)}}{|z-y+x-w|} .
$$

This estimator is location-invariant and unbiased. A numerical estimate of the variance is 1.90 . The distribution is more heavily skewed than log-normal; the hypothesis of normality was rejected by the Anderson-Darling test on $\ln$ (MLE) in about 140 out of 500 runs at the $5 \%$ level of significance.

For the same four observations, let the logarithms of their absolute values $(a, b, c$ and $d)$ be sorted $a<b<c<d$. The $\log \operatorname{HLE}(\sigma)$ is expressed as

$$
\log \operatorname{HLE}(\sigma)= \begin{cases}(b+3 c) / 4, & a+d>2 c \\ (a+b+c+d) / 4, & 2 b<a+d<2 c \\ (3 b+c) / 4, & a+d<2 b\end{cases}
$$

The lower boundary of the variance of the $\log \operatorname{HLE}(\sigma)$ in this case is $\pi^{2} / 16$. The back-transformed estimator, $\operatorname{HLE}(\sigma)$, is positively biased. The asymptotic estimation of the bias correction factor is $\sqrt{\exp \left(\pi^{2} / 16\right)}=1.366$, which is close to our numerical estimate of 1.35. The lower boundary of the variance of $\operatorname{HLE}(\sigma=1)$ is 1.581, which is close to a numerical estimate of 1.70 from our simulations. In our computational study, the log-normality was only rejected in about 50 from 500 runs at the $5 \%$ significance level. 
The $\operatorname{HLEM}(\sigma)$ is also positively, but less, biased. In our study, we estimated the bias correction factor as 1.190. The variance of the estimator is larger than that of $\operatorname{HLE}(\sigma)$, a numerical estimate is 2.90 . The log-normality was rejected in about 120 out of 500 runs at the $5 \%$ significance level.

The $\operatorname{MLE}(\sigma)$ and $\operatorname{HLE}(\sigma)$ are positively correlated but by no means identical. The estimated Pearson coefficients of correlations are about 0.82 for both sample sizes of 3 and 4 . However, as the exact distributions are very skewed, we also estimated the Spearman coefficients, which are about 0.70 and 0.75 correspondingly.

To re-iterate, in the case of known location parameter, for samples $n=3$ and $n=4$, the $\operatorname{HLE}(\sigma)$ is less variable than the $\operatorname{MLE}(\sigma)$. The distribution of $\operatorname{HLE}(\sigma)$ follows reasonably well a log-normal distribution. In contrast, the distribution of the MLE for $n=3$ does not resemble a log-normal distribution and is ill-behaved, with occasional extreme values.

For larger samples, the performances of MLE, HLE and HLEM are comparable. We estimated the parameters of the MLE numerically as the averages of the corresponding parameters acquired in 100 runs of 1000 random and centred Cauchy samples. For each sample, the MLE was found with the nlminb() function in $\mathrm{R}$, the tolerance limit for the objective log-likelihood function was set up at $10^{-3}$. The parameters of HLE and the bias-corrected HLE were also estimated in a similar computational study (100 runs of 1000 random samples each), where $\operatorname{logHLE}$ was estimated as the median (median() function in R) of the upper triangle of the cross-product matrix (outer() function in R) of the logarithms of the absolute values. In Table 1, we show the variance and expected value of the $\operatorname{HLE}(\sigma), \operatorname{MLE}(\sigma)$ and the bias-corrected estimator, i.e. $\operatorname{HLE}(\sigma) /$ (bias factor), for the true scale parameter of $\sigma=1$ and $\sigma=3$, and the location parameter $\mu=0$. The agreement betweent the HLE and MLE is good even for samples as small as 20 .

The parameters of the median-adjusted estimator were derived in a similar computational study and are shown in Table 2 for evensize samples from $C(0,1)$ and $C(0,3)$. The same parameters were obtained for $C(3,1)$ and $C(3,3)$ (as would be expected) and are thus not presented here. The estimator is less biased and slightly more 
variable for $n<20$ in comparison to the other estimators.

\section{Example and conclusion}

In order to illustrate the estimation procedure, we use the example of individual deviations from the mean of 15 observations of the vertical semi-diameter of Venus given in [24]: $(-0.30,+0.48,+0.63,-0.22$, $+0.18,-0.44,-0.24,-0.13,-0.05,+0.39,+1.01,+0.06,-1.40,+0.20$, $+0.10)$.

The maximum likelihood estimator of scale found with nlminb() function in R2.7.2 is 0.2614. Assuming its log-normality and using the estimate of the variance for this sample size for $\sigma=1$ from Table 1, 0.172, the approximate $95 \%$ confidence interval of the true scale parameter is given by the $2.5 \%$ and $97.5 \%$ percentiles of the lognormal $(\ln (0.2614), \ln (0.172+1))$ distribution. This $95 \%$ confidence interval is $(0.1106,0.5271)$. Using the asymptotic variance of $2 / 15$ for the log-normal distribution of MLE, lognormal $(\ln (0.2614), 2 / 15)$, we estimate an asymptotic $95 \%$ confidence interval to be $(0.1278,0.5347)$. Finally, referring to a table of exact percentiles in [10] (Table 3, the location parameter is known), the $95 \%$ confidence interval estimate is $(0.1268,0.5526)$. As we can see, the widths of the estimates based on both the exact percentiles in [10] and our estimation of the exact variance are close, with the both intervals being only about $3 \%$ wider than the asymptotic estimate.

The Hodges-Lehmann estimator on the logarithms of the absolute values of the data in the example is $\log \operatorname{HLE}(\sigma)=-1.3443$. The backtransformed HLE of scale is thus 0.2607. Assuming that the estimator is log-normal and using the exact variance from Table 1, the approximate $95 \%$ confidence interval is $(0.1086,0.5311)$. The asymptotic $95 \%$ confidence interval (assuming the variance of logHLE is $2 /(0.986 n)$ ) is $(0.1268,0.5360)$. The approximate confidence interval for HLE overlaps with the exact confidence interval for MLE, and is only 1.5\% wider. The asymptotic confidence intervals for MLE and HLE are very similar.

The median of the sample is an observed value of 0.06 ; the median of the remaining observations is 0.025 . The median-adjusted HLE is 
0.2656. With the conservative estimate of the variance for $n=14$ from Table 2 and assuming log-normality, the approximate $95 \%$ confidence interval is $(0.1060,0.5563)$. Referring to the table of exact percentiles for the case of unknown location parameter in [10] (Table 4), the 95\% confidence interval based on the MLE $=0.2614$ is $(0.1327,0.6254)$. The interval based on logHLEM is $4 \%$ narrower.

As we can see the point estimates as well as the confidence intervals are very similar for all three estimation procedures (MLE, HLE and HLEM), and the estimates based on the HLE and HLEM are comparable to their MLE counterparts.

In this paper, we discussed the option of using the common HodgesLehmann estimation procedure for estimating the scale parameter of Cauchy distribution. When the location parameter is not known, we suggest adjusting the data by the sample median prior to conducting the computations for the scale estimation. We showed here that the Hodges-Lehmann estimator is asymptotically fully efficient, lognormally distributed and comparable to the maximum likelihood estimator for small and large samples of scale whether the location parameter is known or not. The asymptotic efficiency of the estimator is more than $98 \%$.

The results reported in this paper are of interest for researchers working with Cauchy and hyperbolic secant distributions or in the area of rank estimators.

\section{References}

[1] B.C. Arnold and P.L. Brockett. On distributions whose component ratios are Cauchy. The American Statistician, 46(1):25-26, 1992.

[2] P. Besbeas and B.J.T. Morgan. Integrated squared error estimation of Cauchy parameters. Statistics and Probability Letters, $55: 397-401,2001$.

[3] D.D. Boos. Minimum distance estimators for location and goodness of fit. Journal of the American Statistical Association, 76:663-670, 1981. 
[4] C.L. Brown, R.F. Brcich, and Taleb. A. Suboptimal robust estimation using rank score functions. ICASSP, 1:753-756, 2003.

[5] L.K. Chan. Linear estimation of the location and scale parameters of the Cauchy distribution based on sample quantiles. Journal of the American Statistical Association, 65(330):851-859, 1970.

[6] J.B. Copas. On the unimodality of the likelihood for the Cauchy distribution. Biometrika, 62(3):701 - 704, 1975.

[7] H.A. David. Order statistics. Wiley, NY, USA, 2 edition, 1981.

[8] J. Durbin and M. Knott. Components of Cramer-von Mises statistics. Part I. Journal of the Royal Statistical Society. Series B., 34(2):290-307, 1972.

[9] T.S. Ferguson. Maximum likelihood estimates of the parameters of the Cauchy distribution for samples of size 3 and 4 . Journal of the American Statistical Association, 73:211-213, 1977.

[10] G. Haas, L. Bain, and C. Antle. Inference for the Cauchy distribution based on maximum likelihood estimators. Biometrika, 57(2):403-408, 1970.

[11] T.P. Hettmansperger and J.W. McKean. Robust nonparametrical statistical methods. Kendall's library of Statistics. Arnold, London, UK, 1998.

[12] J.J. Higgins and D.M. Tichenor. Window estimates of location and scale with application to the Cauchy distribution. Applied Mathematics and Computation, 3:113-126, 1977.

[13] J.J. Higgins and D.M. Tichenor. Efficiencies of window estimates of parameters of the Cauchy distribution. Applied Mathematics and Computation, 4:157-165, 1978.

[14] J.L Hodges and E.L. Lehmann. Estimates of location based on rank tests. Annals of Mathematical Statistics, 34:598-611, 1963.

[15] H.A. Howlader and G. Weiss. On Bayesian estimation of the Cauchy parameters. Sankhya Ser. B, 50(3):50-361, 1988.

[16] I.A. Koutrouvelis. Estimation of location and scale in Cauchy distribution using the empirical characteristic function. Biometrika, 69(1):205-213, 1982.

[17] O.Y. Kravchuk. Rank test of location optimal for hyperbolic secant distribution. Comm. Statist. Theory Methods, 34(7):16171630, 2005. 
[18] E.B. Manoukian and P. Nadeau. A note on the hyperbolic secant distribution. American Statistian, 42(1):77-79, 1988.

[19] K.V. Mardia, H.R. Southworth, and C.C. Taylor. On bias in maximum likelihood estimators. Journal of Statistical Planning and Inference, 76:31-39, 1999.

[20] M. Matsui and A. Takemura. Empirical characteristic function approach to goodness-of-fit tests for Cauchy distribution with parameters estimated by MLE or EISE. Annals of Institute of Statistical Mathematics, 57(1):183-199, 2005.

[21] P. McCullagh. Mobius transformation and Cauchy parameter estimation. Annals of Statistics, 24:787-808, 1996.

[22] B.H. Onen, D.C. Dietz, V.C. Yen, and A.H. Moore. Goodnessof-fit tests for the Cauchy distribution. Computational Statistics, 16:97-107, 2001.

[23] R Development Core Team. R: A Language and Environment for Statistical Computing. R Foundation for Statistical Computing, Vienna, Austria, 2008. ISBN 3-900051-07-0.

[24] F. Rublik. A quantile goodness-of-fit test for Cauchy distribution, based on extreme order statistics. Applications of Mathematics, 46:339-351, 2001.

[25] A.K. Saleh, K.M. Hassanein, and E.F. Brown. Optimum spacings for the joint estimation and tests of hypothesis of location and scale parameters of the Cauchy distribution. Comm. Statist. Theory Methods, 14(1):247-254, 1985.

[26] D.C. Vaughan. On the Tiku-Suresh method of estimation. Comm. Statist. Theory Methods, 21(2):451 - 459, 1992.

[27] D.C. Vaughan. The generalized secant hyperbolic distribution and its properties. Comm. Statist. Theory Methods, 31(2):219$238,2002$. 


\begin{tabular}{lccc|ccc}
\hline & \multicolumn{4}{c|}{$C(0,1)$} & \multicolumn{3}{c}{$C(0,3)$} \\
size, $n$ & $\operatorname{HLE}(\sigma)$ & $\operatorname{MLE}(\sigma)$ & $\operatorname{HLE}(\sigma) / \mathrm{bc}$ & $\operatorname{HLE}(\sigma)$ & $\operatorname{MLE}(\sigma)$ & HLE $(\sigma) / \mathrm{bc}$ \\
\hline 10 & $1.115 / 0.314$ & $1.110 / 0.302$ & $1.009 / 0.257$ & $3.346 / 2.810$ & $3.336 / 2.725$ & $3.027 / 2.301$ \\
11 & $1.102 / 0.270$ & $1.102 / 0.269$ & $1.006 / 0.224$ & $3.313 / 2.456$ & $3.295 / 2.348$ & $3.025 / 2.047$ \\
12 & $1.094 / 0.240$ & $1.091 / 0.233$ & $1.006 / 0.203$ & $3.282 / 2.162$ & $3.273 / 2.101$ & $3.020 / 1.830$ \\
13 & $1.086 / 0.216$ & $1.083 / 0.208$ & $1.006 / 0.185$ & $3.259 / 1.947$ & $3.251 / 1.885$ & $3.018 / 1.669$ \\
14 & $1.079 / 0.195$ & $1.078 / 0.191$ & $1.005 / 0.169$ & $3.240 / 1.760$ & $3.233 / 1.717$ & $3.016 / 1.526$ \\
15 & $1.074 / 0.178$ & $1.071 / 0.172$ & $1.004 / 0.156$ & $3.218 / 1.603$ & $3.209 / 1.538$ & $3.011 / 1.403$ \\
20 & $1.054 / 0.124$ & $1.052 / 0.121$ & $1.002 / 0.112$ & $3.160 / 1.140$ & $3.158 / 1.087$ & $3.006 / 1.008$ \\
25 & $1.042 / 0.094$ & $1.040 / 0.093$ & $1.002 / 0.088$ & $3.126 / 0.857$ & $3.125 / 0.831$ & $3.004 / 0.791$ \\
30 & $1.035 / 0.077$ & $1.034 / 0.075$ & $1.001 / 0.072$ & $3.105 / 0.695$ & $3.105 / 0.684$ & $3.004 / 0.650$ \\
35 & $1.030 / 0.065$ & $1.028 / 0.063$ & $1.001 / 0.061$ & $3.090 / 0.583$ & $3.086 / 0.571$ & $3.002 / 0.550$ \\
40 & $1.026 / 0.056$ & $1.027 / 0.055$ & $1.001 / 0.053$ & $3.080 / 0.504$ & $3.076 / 0.489$ & $3.003 / 0.479$ \\
50 & $1.021 / 0.044$ & $1.020 / 0.043$ & $1.001 / 0.042$ & $3.062 / 0.395$ & $3.064 / 0.387$ & $3.002 / 0.379$ \\
60 & $1.017 / 0.036$ & $1.017 / 0.035$ & $1.000 / 0.035$ & $3.052 / 0.325$ & $3.052 / 0.319$ & $3.002 / 0.315$ \\
80 & $1.014 / 0.026$ & $1.014 / 0.026$ & $1.000 / 0.026$ & $3.040 / 0.239$ & $3.038 / 0.234$ & $3.001 / 0.233$ \\
100 & $1.010 / 0.021$ & $1.010 / 0.021$ & $1.000 / 0.021$ & $3.030 / 0.190$ & $3.030 / 0.186$ & $3.000 / 0.186$
\end{tabular}

Table 1: The expected value/variance of the biased HLE and MLE and bias-corrected HLE/(bias correction factor) for samples from centred Cauchy distributions $C(0,1)$ and $C(0,3)$. The simulations were performed in the R-project, R2.7.2; each parameter in the table is the average of 100,000 corresponding estimators. 

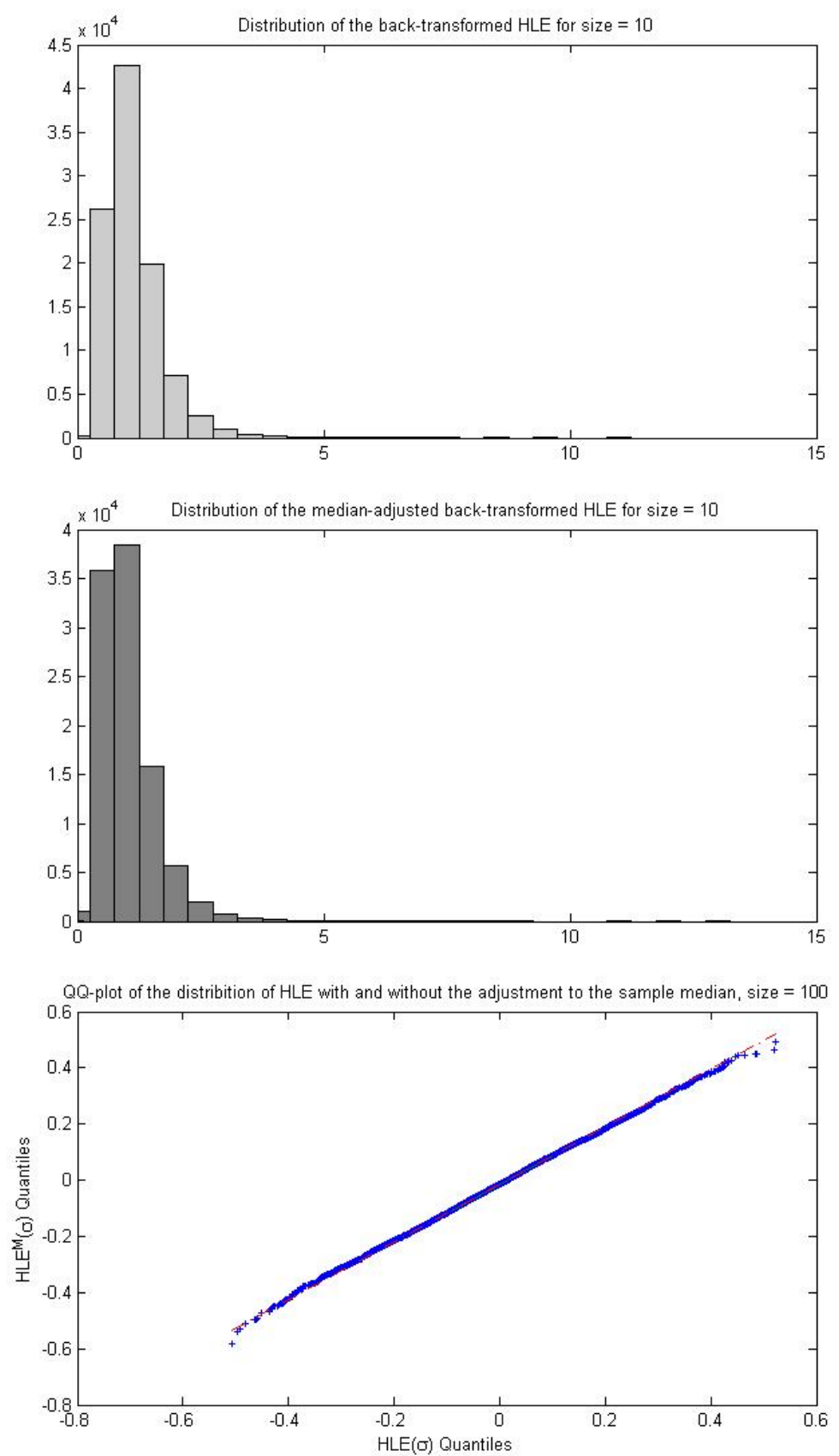

Figure 1: Exact distributions of the back-transformed HLE (upper histogram) and median-adjusted HLE (lower histogram) for samples of 10 observations, and the QQplot of the exact distributions of $\operatorname{HLE}(\sigma)$ and $\operatorname{HLEM}(\sigma)$ for samples of 100 observations $(100,000$ samples from $C(0,1)$ generated with $\operatorname{rcauchy}()$ in $\mathrm{R} 2.7 .2)$. 


\begin{tabular}{lc|c} 
size, $n$ & \multicolumn{2}{c}{ Expected value/variance } \\
& $\sigma=1$ & $\sigma=3$ \\
\hline 10 & $1.012 / 0.326$ & $3.039 / 2.932$ \\
12 & $1.004 / 0.243$ & $3.013 / 2.191$ \\
14 & $1.001 / 0.196$ & $2.999 / 1.755$ \\
20 & $0.997 / 0.124$ & $2.990 / 1.107$ \\
30 & $0.996 / 0.077$ & $2.989 / 0.686$ \\
40 & $0.997 / 0.056$ & $2.989 / 0.497$ \\
50 & $0.996 / 0.044$ & $2.991 / 0.392$ \\
60 & $0.997 / 0.036$ & $2.991 / 0.321$ \\
80 & $0.998 / 0.026$ & $2.993 / 0.238$ \\
100 & $0.998 / 0.021$ & $2.995 / 0.189$
\end{tabular}

Table 2: Estimated expected value and variance of the median-adjusted $\operatorname{HLEM}(\sigma)$ for small and large samples from Cauchy $C(0,1)$ and $C(0,3)$ (100,000 runs of rcauchy() in R2.7.2). 